\title{
Dual-Target Binding Ligands with Modulated Pharmacokinetics for Endoradiotherapy of Prostate Cancer
}

\author{
James M. Kelly ${ }^{1}$, Alejandro Amor-Coarasa ${ }^{1}$, Anastasia Nikolopoulou ${ }^{1}$, Till Wüstemann ${ }^{1}$, Peter Barelli²,3, Dohyun Kim ${ }^{4}$, \\ Clarence Williams Jr. ${ }^{1}$, Xiwei Zheng ${ }^{5}$, Cong Bi ${ }^{5}$, Bao Hu${ }^{6}$, J. David Warren ${ }^{2,3,7}$, David S. Hage ${ }^{5}$, Stephen G. DiMagno ${ }^{6}$, \\ and John W. Babich ${ }^{1,3,7}$ \\ ${ }^{I}$ Division of Radiopharmaceutical Sciences and $\mathrm{MI}^{3}$, Department of Radiology, Weill Cornell Medicine, New York, New York; \\ ${ }^{2}$ Abby and Howard P. Milstein Synthetic Chemistry Core Facility, Weill Cornell Medicine, New York, New York; ${ }^{3}$ Department of \\ Biochemistry, Weill Cornell Medicine, New York, New York; ${ }^{4}$ Citigroup Biomedical Imaging Center, Weill Cornell Medicine, \\ New York, New York; ${ }^{5}$ Department of Chemistry, University of Nebraska-Lincoln, Lincoln, Nebraska; ${ }^{6}$ Departments of Medicinal \\ Chemistry and Pharmacognosy \& Chemistry, University of Illinois at Chicago, Chicago, Illinois; and ${ }^{7}$ Sandra and Edward Meyer \\ Cancer Center, Weill Cornell Medicine, New York, New York
}

Prostate-specific membrane antigen (PSMA)-targeted radiotherapy of prostate cancer $(\mathrm{PCa})$ has emerged recently as a promising approach to the treatment of disseminated disease. A small number of ligands have been evaluated in patients, and although early tumor response is encouraging, relapse rate is high and these compounds localize to the parotid, salivary, and lacrimal glands as well as to the kidney, leading to dose-limiting toxicities and adverse events affecting quality of life. We envision that dual-target binding ligands displaying high affinity for PSMA and appropriate affinity for human serum albumin (HSA) may demonstrate a higher therapeutic index and be suitable for treatment of PCa by targeted $\alpha$-therapy. Methods: Six novel urea-based ligands with varying affinities for PSMA and HSA were synthesized, labeled with ${ }^{131} \mathrm{I}$, and evaluated by in vitro binding and uptake assays in LNCaP cells. Four compounds were advanced for further evaluation in a preclinical model of PCa. The compounds were compared with MIP-1095, a PSMA ligand currently in clinical evaluation. Results: The compounds demonstrated affinity for PSMA on the order of $4-40 \mathrm{nM}$ and affinity for HSA in the range of $1-53 \mu \mathrm{M}$. Compounds with relatively high affinity for HSA $(\leq 2 \mu \mathrm{M})$ showed high and sustained blood-pool activity and reduced uptake in the kidneys. ${ }^{131}$ I-RPS-027, with a $50 \%$ inhibitory concentration (PSMA) of $15 \mathrm{nM}$ and a dissociation constant (HSA) of $11.2 \mu \mathrm{M}$, cleared from the blood over the course of $48 \mathrm{~h}$ and showed good tumor uptake (10 percentage injected dose per gram) and retention and a greater than 5-fold decrease in kidney uptake relative to MIP1095. The tumor-to-kidney ratio of ${ }^{131}$ I-RPS-027 was greater than $3: 1$ at $24 \mathrm{~h}$ after injection, increasing to $7: 1$ by $72 \mathrm{~h}$. Conclusion: RPS-027 shows dual targeting to PSMA and albumin, resulting in a high tumor uptake, highly favorable tissue distribution, and promising therapeutic profile in a preclinical model of prostate cancer. In comparison to existing ligands proposed for targeted therapy of prostate cancer, RPS-027 has tumor-to-tissue ratios that predict a significant reduction in side effects during therapy. Using iodine/radioiodine as a surrogate for the radiohalogen ${ }^{211} \mathrm{At}$, we therefore propose dual-target binding ligands such as RPS-027 as next-generation radiopharmaceuticals for targeted $\alpha$-therapy using ${ }^{211} \mathrm{At}$.

Received Dec. 16, 2016; revision accepted Apr. 11, 2017

For correspondence or reprints contact: John W. Babich, Department of Radiology, Weill Cornell Medicine, Belfer Research Building, Rm. 1600, 413 East 69th St., New York, NY 10021

E-mail: job2060@med.cornell.edu

Published online Apr. 27, 2017.

COPYRIGHT (c) 2017 by the Society of Nuclear Medicine and Molecular Imaging.
Key Words: prostate cancer; PSMA; albumin; targeted radiotherapy

J Nucl Med 2017; 58:1442-1449

DOI: 10.2967/jnumed.116.188722

$\mathbf{T}$ argeted radiotherapy (TRT), the systemic application of radiolabeled drugs or biologicals to treat disseminated cancers, has reached a tipping point with the pending approval of Lutathera $\left({ }^{177} \mathrm{Lu}-\right.$ DOTATATE; Advanced Accelerator Applications Inc.) for the treatment of neuroendocrine cancer (1) and with the publication of promising clinical results for the treatment of metastatic castrateresistant prostate cancer with radiolabeled small-molecule inhibitors of prostate-specific membrane antigen (PSMA) (2-8). These low-molecular-weight peptidomimetic structures rapidly distribute throughout the body and clear rapidly from the blood (9). In contrast to larger targeting constructs such as immunoglobulins, the low molecular weight facilitates capillary transport and penetration into solid tumors $(10,11)$. Such rapid and systemic tissue access is desirable for targeting of tumors and enabling access to widely disseminated tumor lesions (12). However, rapid tissue access and distribution may also enable off-target interactions in normal tissues that express physiologic levels of the target or may lead to concentration of the therapeutic radiopharmaceutical in excretory organs such as the kidney. These interactions may lead to normal tissues receiving radiation doses that cause potentially fatal irreversible tissue damage (13-15).

To date, TRT of prostate cancer with low-molecular-weight PSMA inhibitors has not required nephroprotection schemes despite demonstrable renal accumulation of the radiotherapeutics, though renal radiation dose must be considered and tracked because modest response rates and high rates of relapse mean that patients receive several cycles of $\beta$-emitting compounds over their course of treatment $(2,3)$. PSMA therapy is associated, however, with concentration of therapeutic radiopharmaceuticals in the lacrimal, salivary, and parotid glands of patients; irradiation of these tissues can cause glandular dysfunction leading to xerostomia or dry eye $(2,3)$. Off-target effects may become severe when patients receive multiple cycles of $\beta$-emitter or in the case when the therapeutic radionuclide chosen is an $\alpha$-emitter (16). 
Preliminary experiments in preclinical models have suggested that uptake in these structures can be reduced by treatment with 2-(phosphonomethyl)-pentandioic acid, a high-affinity PSMA inhibitor $(17,18)$. An alternative to such a pharmacologic displacement strategy is to modulate normal tissue access via compartmentalization of the radiopharmaceutical in safe-haven tissues. The blood pool becomes an attractive space to consider compartmentalizing $\alpha$-emitters because it would allow access to the tumor vascular bed but should hinder rapid diffusion into normal tissues. At any one time, about $60 \%-70 \%$ of the entire blood volume in a human is contained in the veins (19). Hence, an $\alpha$-emitter compartmentalized to the blood pool will reduce offtarget effects because most veins have diameters considerably greater $(200-5,000 \mu \mathrm{m})$ than the $\alpha$-particle length of radionuclides such as ${ }^{211}$ At $(25-100 \mu \mathrm{m})(20)$. Albumin targeting is ideal for such an application because the protein is abundant in blood serum, has a long physiologic half-life, and is known to reversibly bind small negatively charged or hydrophobic molecules (21-23). Reversible binding to albumin has been shown to extend blood clearance and change tissue distribution of biologics (24), peptides (25), drugs (26), contrast agents (27), and radiopharmaceuticals $(23,28)$.

We report herein the results of our efforts to design a new class of dual-target binding small molecules that display high affinity (low nanomolar) chemical targeting to prostate cancer cells through interaction with PSMA, and display a range of moderate (micromolar) affinities for albumin. We show that modulation of albumin affinity among potent PSMA ligands has a demonstrable effect on tumor targeting and normal organ compartmentalization and residence time. To study this phenomenon, we use iodine/ radioiodine as a surrogate for the $\alpha$-emitting radiohalogen ${ }^{211} \mathrm{At}$ (29-31), and our results support investigation of such dual-target binding ligands as next-generation radiopharmaceuticals for $\alpha$-targeted therapy of prostate cancer using ${ }^{211} \mathrm{At}$.

\section{MATERIALS AND METHODS}

\section{Chemistry}

A full description of the synthesis of compounds RPS-001, RPS005, RPS-020, RPS-022, RPS-023, RPS-025, RPS-026, and RPS027 (Fig. 1) and the corresponding organostannane precursors 11-17 (the organostannanyl analog of RPS-026 was not prepared) can be found in the supplemental materials (available at http://jnm. snmjournals.org).

\section{Radiosynthesis}

Radiolabeling was performed as previously described with modification (2). One hundred microliters of a $250 \mu \mathrm{g} / \mathrm{mL}$ solution of organnostannane precursor 11-17 in EtOH was added to a vial containing $74-740 \mathrm{MBq}(2-20 \mathrm{mCi})$ of $\mathrm{Na}^{124} \mathrm{I}$ or $\mathrm{Na}^{131} \mathrm{I}$ in $30-60 \mu \mathrm{L}$ of aqueous $\mathrm{NaOH}$. A $15 \% \mathrm{v} / \mathrm{v}_{2} \mathrm{O}_{2} / \mathrm{AcOH}$ solution was prepared, and $50 \mu \mathrm{L}$ were transferred immediately to the reaction vial. The reaction was mixed for $20 \mathrm{~s}$ and let stand for $5 \mathrm{~min}$ at room temperature. It was then diluted with $3 \mathrm{~mL}$ of $\mathrm{H}_{2} \mathrm{O}$ and passed through a preactivated SOLA cartridge (Thermo Scientific). The cartridge was washed with $\mathrm{H}_{2} \mathrm{O}(3 \mathrm{~mL})$ and dried with air. The radiolabeled intermediate was eluted into a second vial with $1 \mathrm{~mL}$ of a $4 \mathrm{M} \mathrm{HCl} /$ dioxane solution. The reaction was mixed for $20 \mathrm{~s}$ and let stand for $40 \mathrm{~min}$. It was then diluted with $\mathrm{H}_{2} \mathrm{O}(9 \mathrm{~mL})$ and passed through a preactivated Bond Elut Plexa cartridge (Agilent Technologies, Inc.). The cartridge was washed with $5 \mathrm{~mL}$ of a $20 \% \mathrm{v} / \mathrm{v}$ EtOH/ $\mathrm{H}_{2} \mathrm{O}$ solution and dried with air. The radiolabeled product was eluted with dimethyl sulfoxide (100-300 $\mu \mathrm{L})$.

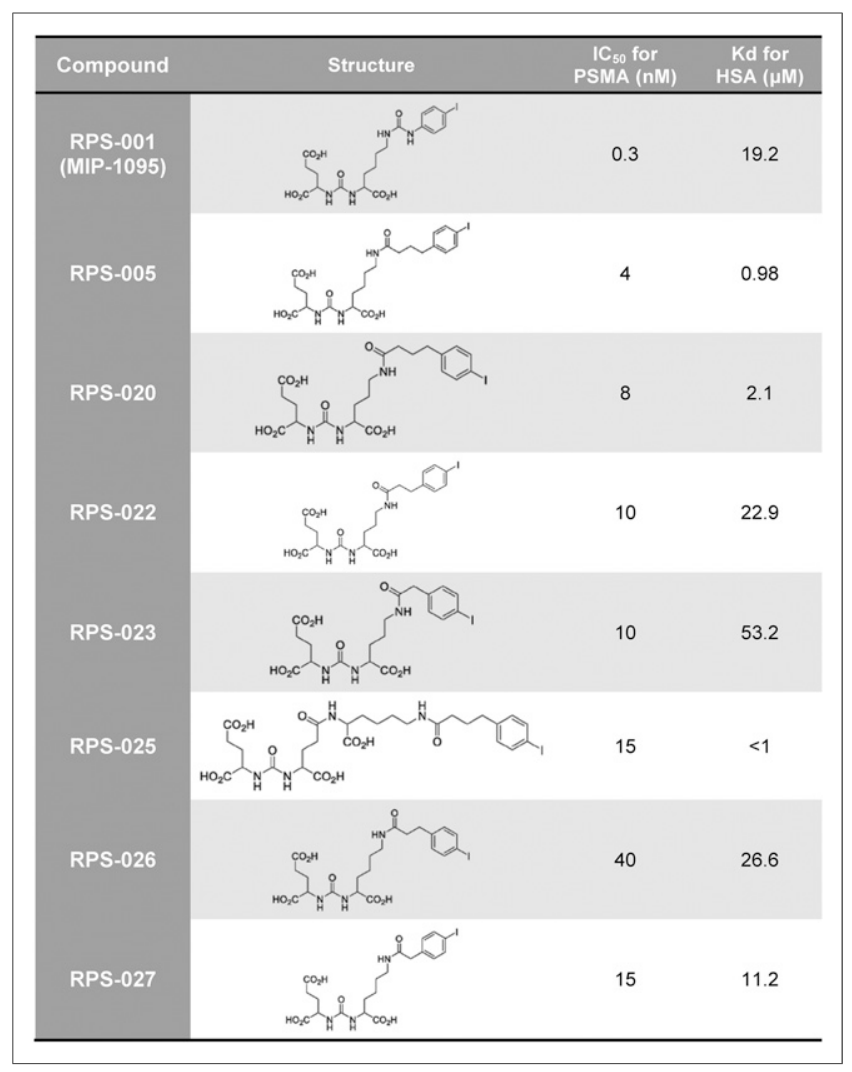

FIGURE 1. Structures of RPS series compounds and their affinities for PSMA and HSA.

\section{Human Serum Albumin (HSA) Affinity Determination}

HSA was immobilized to high-performance liquid chromatographygrade silica by the Schiff base method as described previously and was packed into $10 \times 2.1 \mathrm{~mm}$ inner diameter microcolumns $(32,33)$. The protein content of these columns was approximately $60 \mathrm{mg}$ of HSA per gram of silica (34). Control microcolumns were prepared in the same manner but with no HSA being added during the immobilization step. The retention factor for each compound was measured on both an HSA microcolumn and a control column by injecting $5-\mu \mathrm{L}$ samples that contained approximately $50 \mu \mathrm{M}$ of the compound in $0.067 \mathrm{M}$ potassium phosphate buffer ( $\mathrm{pH}$ 7.4). All samples were injected in triplicate at room temperature and at $1.0 \mathrm{~mL} / \mathrm{min}$, with the phosphate buffer used as the mobile phase. Similar injections were made with samples containing sodium nitrate, which was used as a void volume marker. Elution of the injected compounds was monitored by absorbance detection. The dissociation constant $(\mathrm{Kd})$ for each compound with HSA was determined using the measured retention factors, after correction for any observed retention on the control column, along with the estimated content of active HSA in the column, as based on injections made with warfarin and L-tryptophan (i.e., probes for Sudlow sites I and II of HSA) $(34,35)$. The estimated precision of the Kd values was $\pm 2 \%-14 \%$.

\section{Cell Culture}

The PSMA-expressing human prostate cancer cell line LNCaP was obtained from the American Type Culture Collection. Cell culture supplies were from Invitrogen unless otherwise noted. LNCaP cells were maintained in RPMI-1640 medium supplemented with $10 \%$ fetal bovine serum (Hyclone), $4 \mathrm{mM}$-glutamine, $1 \mathrm{mM}$ sodium pyruvate, $10 \mathrm{mM} \mathrm{N}$-2-hydroxyethylpiperazine- $N$-2-ethanesulfonic acid, $2.5 \mathrm{mg} / \mathrm{mL}$ D-glucose, and $50 \mu \mathrm{g} / \mathrm{mL}$ gentamicin in a humidified incubator at 
$37^{\circ} \mathrm{C} / 5 \% \mathrm{CO}_{2}$. Cells were removed from flasks for passage or for transfer to 12 -well assay plates by incubating them with $0.25 \%$ trypsin/ethylenediaminetetraacetic acid.

\section{In Vitro Determination of $\mathbf{5 0 \%}$ Inhibitory Concentration $\left(\mathbf{I C}_{\mathbf{5 0}}\right)$}

$\mathrm{IC}_{50}$ values of the nonradioactive iodine-containing ligands were determined by screening in a multiconcentration competitive binding assay against $99 \mathrm{~m} \mathrm{Tc}-((7 S, 12 S, 16 S)-1$-(1-(carboxymethyl)- $1 H$-imidazol2-yl)-2-((1-(carboxymethyl)-1H-imidazol-2-yl)methyl)-9,14-dioxo2,8,13,15-tetraazaoctadecane-7,12,16,18-tetracarboxylic acid technetium tricarbonyl complex ( ${ }^{99 \mathrm{~m} T c-M I P-1427)}$ for binding to PSMA on LNCaP cells, according to methods previously described (36). Briefly, LNCaP cells were plated $48 \mathrm{~h}$ before the experiment to achieve a density of approximately $5 \times 10^{5}$ cells/well (in triplicate) in RPMI-1640 medium supplemented with $0.25 \%$ bovine serum albumin. The cells were incubated for $1 \mathrm{~h}$ with $1 \mathrm{nM}$ 99mTc-MIP-1427 in serum-free RPMI-1640 medium in the presence of $0.1-10,000 \mathrm{nM}$ test compounds. Radioactive incubation medium was then removed by pipette, and the cells were washed twice using $1 \mathrm{~mL}$ of ice-cold $\mathrm{N}$-2-hydroxyethylpiperazine- $\mathrm{N}$-2ethanesulfonic acid buffer. Cells were harvested from the plates and transferred to tubes for radioactive counting using a Packard Cobra II $\gamma$-Counter. $\mathrm{IC}_{50}$ values were determined by nonlinear regression using GraphPad Prism software (GraphPad Software).

\section{Inoculation of Mice with Xenografts}

All animal studies were approved by the Institutional Animal Care and Use Committee of Weill Cornell Medicine and were undertaken in accordance with the guidelines set forth by the U.S. Public Health Service Policy on Humane Care and Use of Laboratory Animals. Animals were housed under standard conditions in approved facilities with 12-h light-dark cycles. Food and water were provided ad libitum throughout the course of the studies. Male inbred athymic $n u / n u$ mice were purchased from The Jackson Laboratory. For inoculation in mice, $\mathrm{LNCaP}$ cells were suspended at $4 \times 10^{7}$ cells $/ \mathrm{mL}$ in a $1: 1$ mixture of phosphate-buffered saline:Matrigel (BD Biosciences). Each mouse was injected in the left flank with $0.25 \mathrm{~mL}$ of the cell suspension. The mice were imaged when the tumors reached approximately $200-400 \mathrm{~mm}^{3}$, whereas biodistributions were conducted when tumors were in the range $100-400 \mathrm{~mm}^{3}$.

\section{Tissue Distribution Studies}

A quantitative analysis of the tissue distribution of ${ }^{131}$ I-labeled compounds was performed in separate groups of male $\mathrm{NCr}-n u / n u$ mice bearing $\mathrm{LNCaP}$ cell xenografts administered via the tail vein as a bolus injection $(\sim 370 \mathrm{kBq}[10 \mu \mathrm{Ci}] /$ mouse $)$ in a volume of $0.05-0.1 \mathrm{~mL}$ of saline solution containing $2.5 \% \mathrm{v} / \mathrm{v}$ dimethyl sulfoxide. The animals ( $n=3-5 /$ time point) were euthanized by asphyxiation with $\mathrm{CO}_{2}$ at the indicated time points after injection. Tissues, including blood, heart, lungs, liver, spleen, pancreas, kidneys, stomach, large and small intestines (with contents), skeletal muscle, bone, and tumor, were dissected, excised, weighed wet (Sartorius analytic balance), and counted in a Wizard automated $\gamma$-counter (Perkin Elmer). A 1 percentage injected dose per gram (\%ID/g) standard was counted with the tissue samples. Tissue time-radioactivity levels expressed as \% ID/g were determined. Blood pharmacokinetics were modeled as a dual-compartment system using biexponential least-squares regression fit to the data. The regression method was implemented in MATLAB R2015b (The MathWorks). An independent-samples $t$ test was used to compare different time points and between different compounds, and in all analyses the statistical significance $(\alpha$-level) was set at a $P$ value of less than 0.05 .

\section{Imaging Studies}

LNCaP xenograft tumor-bearing mice ( 2 per compound) were injected intravenously via the tail vein as a bolus injection of 7.03-7.77 MBq
(190-210 $\mu \mathrm{Ci}$ ) of ${ }^{124}$ I-RPS-027. The specific activity of ${ }^{124}$ I-RPS-027 was in the range of $3-10 \mathrm{GBq} / \mu \mathrm{mol}$. The mice were imaged by small-animal PET/CT (Inveon; Siemens Medical Solutions, Inc.) at 1, 3, 6, 24, and $48 \mathrm{~h}$ after injection. Total acquisition time was $30 \mathrm{~min}$, and a CT scan was obtained either immediately before or immediately after the acquisition for both anatomic coregistration and attenuation correction. The data were reconstructed using the commercial Inveon software supplied by the vendor. Image-derived tumor uptake was estimated by drawing a region of interest.

\section{RESULTS}

\section{Chemistry and Radiochemistry}

Reference compounds and their trimethylstannyl precursors were synthesized as described in Supplemental Figure 1. Synthesis yields ranged from $4 \%$ to $20 \%$ in $5-8$ steps from L-Glu(OtBu)$\mathrm{OtBu} \cdot \mathrm{HCl}$. Trimethylstannylation was accomplished in $20 \%-82 \%$ yield from the protected 4-iodophenyl precursors.

Radiolabeling was accomplished by an iododestannylation reaction, followed by deprotection of the labeled intermediate in acidic conditions (Fig. 2). Total reaction time was $60 \mathrm{~min}$, radiochemical yield ranged from $43 \%$ to $72 \%$, and radiochemical purity was greater than $90 \%$ for all compounds tested. Specific activity varied from 2 to $10 \mathrm{GBq} / \mu \mathrm{mol}$ according to the starting ${ }^{124} \mathrm{I}$ or ${ }^{131} \mathrm{I}$ activity. The deprotection step proved to be time sensitive: for reaction times below $40 \mathrm{~min}$, incomplete deprotection was observed, whereas reaction times greater than $45 \mathrm{~min}$ led to the formation of an unidentified impurity that could not be removed during purification by solid-phase extraction. The characteristics of the radiosyntheses are described in Table 1. No significant difference in labeling yield was observed when ${ }^{124} \mathrm{I}$ was used in place of ${ }^{131} \mathrm{I}$.

\section{In Vitro Studies}

The range of affinities for PSMA was determined to be 4-40 nM, with most of the compounds clustered between 10 and $15 \mathrm{nM}$ (Fig. 1). In the same assay RPS-001, also known as MIP-1095, was found to have an $\mathrm{IC}_{50}$ of $0.3 \mathrm{nM}$. Compounds bearing the $p$-(iodophenyl) butyric acid moiety were found to have a high affinity $(1-2 \mu \mathrm{M})$ for HSA (Fig. 1). RPS-025 could not be eluted from the column, which prevented the calculation of a precise Kd. The compound RPS-027 had a modest affinity of $11 \mu \mathrm{M}$, whereas RPS-001, RPS-022, and RPS-026 were in the range 19-26 $\mu \mathrm{M}$. RPS-023 ( $\mathrm{Kd}=53.2 \mu \mathrm{M})$ was determined to have a weak affinity.

\section{Biodistribution and Small-Animal PET/CT Imaging}

The biodistribution studies of the 6 ligands demonstrated that albumin binding affinity contributed markedly to the different pharmacokinetics observed in mice. RPS-001 ( $\mathrm{Kd}=20 \mu \mathrm{M}$ for

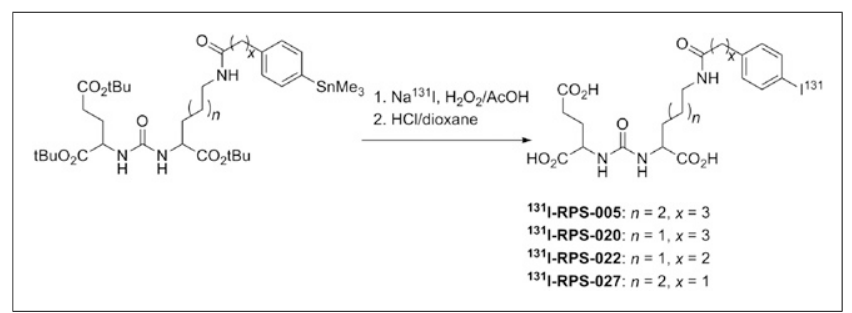

FIGURE 2. Radiosynthesis of dual-target binding ligands via iododestannylation reactions. ${ }^{131}$ I-RPS-001 and ${ }^{131}$ I-RPS-025 were synthesized by same procedure from their corresponding organostannane precursors. 
TABLE 1

Characteristics of Radiosynthesis of Dual-Target Binding Ligands with ${ }^{131}$ |

\begin{tabular}{cccc}
\hline Compound & RCY $(\%)$ & RCP $(\%)$ & SA $(\mathrm{GBq} / \mu \mathrm{mol})^{\star}$ \\
\hline RPS-001 & 71.8 & $>97$ & 3.15 \\
RPS-005 & 62.0 & $>96$ & 3.52 \\
RPS-020 & 66.7 & $>94$ & 4.29 \\
RPS-022 & 53.6 & $>90$ & 3.06 \\
RPS-025 & 43.5 & $>93$ & 2.02 \\
RPS-027 & 49.5 & $>98$ & 9.18
\end{tabular}

*Based on a starting activity of $72-370 \mathrm{MBq}(2-10 \mathrm{mCi})$.

$\mathrm{RCY}=$ radiochemical yield; $\mathrm{RCP}=$ radiochemical purity; $\mathrm{SA}=$ specific activity.

HSA) demonstrated relatively rapid clearance from the blood (Fig. 3A). The measured changes in blood activity became statistically significant by $72 \mathrm{~h}$. Initial kidney uptake was high $(>100 \% \mathrm{ID} / \mathrm{g})$, and from 24 to $96 \mathrm{~h}$ after injection ${ }^{131}$ I-RPS-001 activity in the kidney cleared from $65.24 \pm 22.61 \% \mathrm{ID} / \mathrm{g}$ at $24 \mathrm{~h}$ to $2.12 \pm 2.47 \% \mathrm{ID} / \mathrm{g}$ at $96 \mathrm{~h}$. The measured changes in kidney activity became statistically significant by $48 \mathrm{~h}$. Tumor uptake was high, with gradual tumor washout observed over several days $(19.81 \pm 6.16 \% \mathrm{ID} / \mathrm{g}$ at $24 \mathrm{~h}$ vs. $10.21 \pm 4.30 \% \mathrm{ID} / \mathrm{g}$ at $96 \mathrm{~h})($ Fig. $3 \mathrm{~A})$, such that the tumor-to-kidney ratio increased with time. Low, PSMA-mediated uptake was observed at $24 \mathrm{~h}$ after injection in the large intestine $(2.35 \pm 1.26 \% \mathrm{ID} / \mathrm{g})$ and the spleen $(2.41 \pm 1.40 \% \mathrm{ID} / \mathrm{g})$, and accumulated activity was negligible in all tissues except for the tumor and kidney by $48 \mathrm{~h}$ after injection. The tissue uptake at earlier time points was extrapolated using previously reported data (37).

Accumulation of ${ }^{131} \mathrm{I}$-RPS-005 ( $\mathrm{Kd}=0.89 \mu \mathrm{M}$ for HSA) in the blood was exceptionally high, and clearance was slow and statistically insignificant over the 96-h observation window (Fig. 3B). At $24 \mathrm{~h}$ after injection, blood activity was $21.35 \pm 3.99 \% \mathrm{ID} / \mathrm{g}$, which decreased to $15.57 \pm 3.98 \% \mathrm{ID} / \mathrm{g}$ by $96 \mathrm{~h}$. This slow blood clearance is likely to be responsible for the non-PSMA-mediated uptake observed in tissues such as the heart $(4.40 \pm 0.74 \% \mathrm{ID} / \mathrm{g}$ at $24 \mathrm{~h})$, lungs $(8.87 \pm 0.74 \% \mathrm{ID} / \mathrm{g}$ at $24 \mathrm{~h})$, and liver $(3.41 \pm 0.56$
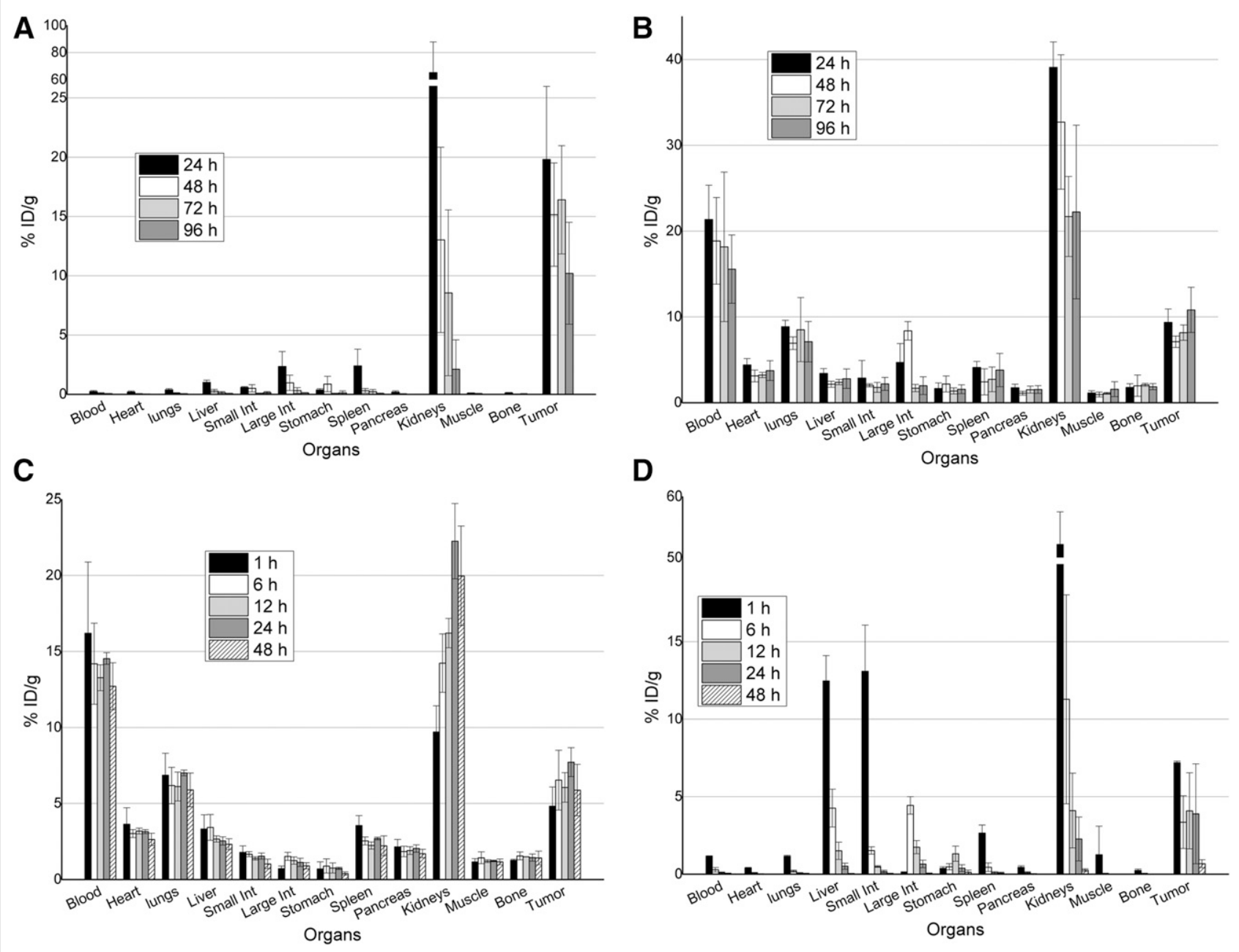

FIGURE 3. Biodistribution of dual-target binding ligands in nude mice bearing PSMA+ LNCaP human tumor xenografts. Error bars represent SD of measured values. (A) ${ }^{131} \mathrm{I}$-RPS-001 at $24,48,72$, and $96 \mathrm{~h}$ after injection. (B) ${ }^{131} \mathrm{I}-\mathrm{RPS}-005$ at $24,48,72$, and $96 \mathrm{~h}$ after injection. (C) ${ }^{131} \mathrm{I}-\mathrm{RPS}-020$ at $1,6,12,24$, and $48 \mathrm{~h}$ after injection. (D) ${ }^{131} \mathrm{I}$-RPS-022 at 1, 6, 12, 24, and $48 \mathrm{~h}$ after injection. Int = intestine. 


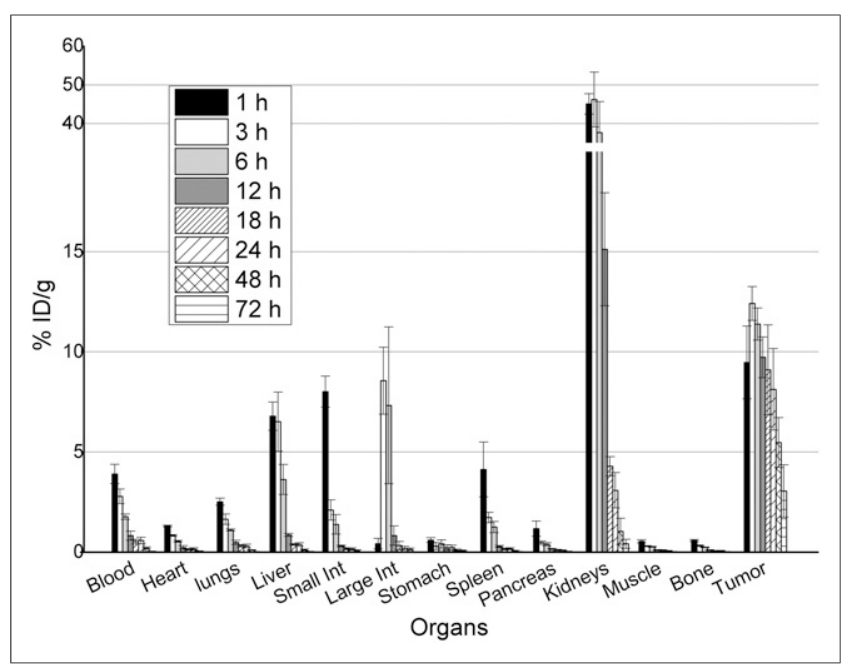

FIGURE 4. Biodistribution of ${ }^{131}$ I-RPS-027 in nude mice bearing PSMA+ LNCaP human tumor xenografts. Error bars represent SD of measured values. Int $=$ intestine.

$\%$ ID/g at $24 \mathrm{~h})$. Kidney uptake (39.09 $\pm 2.96 \% \mathrm{ID} / \mathrm{g})$ was lower at $24 \mathrm{~h}$ after injection than observed for ${ }^{131}$ I-RPS-001, but clearance was considerably slower. In combination with the comparatively low tumor uptake of approximately $10 \%(9.37 \pm 1.56 \% \mathrm{ID} / \mathrm{g}$ at $24 \mathrm{~h} ; 10.82 \pm 2.64 \% \mathrm{ID} / \mathrm{g}$ at $96 \mathrm{~h}$ ), these pharmacokinetics result in poor tumor-to-background ratios at all time points.

Because the tissue uptake of ${ }^{131}$ I-RPS-005 appeared to peak within the first $24 \mathrm{~h},{ }^{131} \mathrm{I}$-RPS-020 (Kd $\left.=2.1 \mu \mathrm{M}\right)$ was investigated at early time points as well. Prolonged blood retention was also observed, with an initial accumulation of $16.20 \pm 4.68 \% \mathrm{ID} / \mathrm{g}$ at $1 \mathrm{~h}$ after injection only decreasing to $12.71 \pm 1.55 \% \mathrm{ID} / \mathrm{g}$ at $48 \mathrm{~h}$ (Fig. 3C), which was not statistically significant. This was associated with high off-target uptake, most notably in the lungs $(6.12 \pm 0.95 \% \mathrm{ID} / \mathrm{g}$ at $1 \mathrm{~h}$ after injection) and kidneys $(16.21 \pm$ $0.97 \% \mathrm{ID} / \mathrm{g}$ at $1 \mathrm{~h}$ ). Activity continued to accumulate in the kidneys, peaking at $22.26 \pm 2.48 \% \mathrm{ID} / \mathrm{g}$ at $24 \mathrm{~h}$ after injection. Tumor uptake $(4.81 \pm 1.27 \% \mathrm{ID} / \mathrm{g})$ was lower than observed for RPS-005, as a comparison of PSMA affinities might predict, but with no statistically significant change over the course of $48 \mathrm{~h}$.

In contrast, ${ }^{131} \mathrm{I}$-RPS-022 $(\mathrm{Kd}=22.9 \mu \mathrm{M})$ showed a rapid and significant drop in blood activity over time, with negligible activity detected as early as $12 \mathrm{~h}$ after injection (Fig. 3D). Considerable uptake was observed in the liver $(12.46 \pm 1.63 \% \mathrm{ID} / \mathrm{g})$ and small intestine $(13.09 \pm 2.98 \% \mathrm{ID} / \mathrm{g})$ at $1 \mathrm{~h}$ after injection, though clearance from each organ was rapid. Kidney uptake, which reached $(52.18 \pm 5.35 \% \mathrm{ID} / \mathrm{g})$ at $1 \mathrm{~h}$ after injection, demonstrated

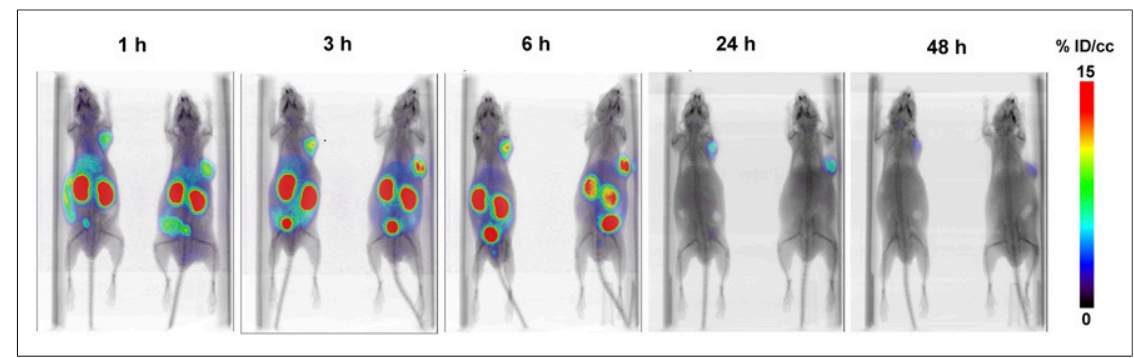

FIGURE 5. Small-animal PET/CT imaging of LNCaP xenograft mice by small-animal PET/CT using ${ }^{124}$ I-RPS-027 (7.4 MBq [200 $\left.\mu \mathrm{Ci}\right]$ ). a significant clearance of activity, reaching $2.27 \pm 1.41 \% \mathrm{ID} / \mathrm{g}$ by $24 \mathrm{~h}$, leading to favorable tumor-to-kidney and tumor-to-background ratios at later time points. However, tumor uptake peaked at $7.20 \pm$ $0.10 \% \mathrm{ID} / \mathrm{g}$ at $1 \mathrm{~h}$ after injection and decreased significantly to $3.35 \pm 1.70 \% \mathrm{ID} / \mathrm{g}$ by $6 \mathrm{~h}$.

${ }^{131}$ I-RPS-027 showed a promising biodistribution profile over the time period studied (Fig. 4). Activity in the blood at $1 \mathrm{~h}$ after injection was $3.91 \pm 0.48 \% \mathrm{ID} / \mathrm{g}$, which decreased to $0.58 \pm 0.17$ $\%$ ID/g by $24 \mathrm{~h}$. Statistically significant decreases in blood activity were observed over the entire observation period. Initial uptake was also observed in the liver $(6.79 \pm 0.70 \% \mathrm{ID} / \mathrm{g} ; 1 \mathrm{~h})$, small intestine $(8.01 \pm 0.78 \% \mathrm{ID} / \mathrm{g} ; 1 \mathrm{~h})$, large intestine $(8.56 \pm 1.67$ $\% \mathrm{ID} / \mathrm{g} ; 3 \mathrm{~h})$, spleen $(4.13 \pm 1.37 \% \mathrm{ID} / \mathrm{g} ; 1 \mathrm{~h})$, and heart $(1.30 \pm$ $0.04 \% \mathrm{ID} / \mathrm{g} ; 1 \mathrm{~h})$. Clearance from these tissues decreased largely in proportion to blood clearance, suggesting that the normal organ activity is related to blood-pool activity rather than tissue uptake. Minimal activity was detected in the tissue by $12 \mathrm{~h}$ after injection with the exception of the kidneys $(15.12 \pm 2.82 \% \mathrm{ID} / \mathrm{g})$ and the tumor $(9.73 \pm 1.01 \% \mathrm{ID} / \mathrm{g})$. Although maximum tumor uptake $(12.41 \pm 0.84 \% \mathrm{ID} / \mathrm{g} ; 3 \mathrm{~h})$ was lower than for ${ }^{131} \mathrm{I}$-RPS-001, tumor uptake remained as high as $8.13 \pm 2.03 \% \mathrm{ID} / \mathrm{g}$ at $24 \mathrm{~h}$ and showed no significant change over $24 \mathrm{~h}$ period but dropped significantly to $3.05 \pm 1.30 \% \mathrm{ID} / \mathrm{g}$ at $72 \mathrm{~h}$, leading to excellent tumor-to-background and tumor-to-kidney $(>2)$ ratios as early as $18 \mathrm{~h}$ after injection. Although tumor uptake of ${ }^{131}$ I-RPS-027 was roughly $50 \%$ of that for ${ }^{131}$ I-RPS-001 at all time points studied, the kidney concentration of ${ }^{131}$ I-RPS-027 was 5-fold less than that for ${ }^{131}$ I-RPS-001.

Desirable pharmacokinetics continued to be observed at longer time points for ${ }^{131} \mathrm{I}$-RPS-027 (Fig. 4). Activity in the blood remained detectable up to $48 \mathrm{~h}$ after injection $(0.20 \pm 0.06$ $\% \mathrm{ID} / \mathrm{g}$ ), whereas tumor-to-normal-tissue ratios continued to increase because of rapid clearance from the kidneys $(1.04 \pm 0.65$ $\% \mathrm{ID} / \mathrm{g}$ at $48 \mathrm{~h}$ ). These in vivo findings were visually recapitulated by small-animal PET/CT imaging of LNCaP xenograft mice using ${ }^{124}$ I-RPS-027. Initial uptake in the tumor, kidneys, and hepatobiliary system is evident at $1 \mathrm{~h}$ (Fig. 5), with clearance from nontarget tissue resulting in highly specific tumor targeting at 24 and $48 \mathrm{~h}$ after injection.

Time-activity curves were plotted to facilitate a greater understanding of the pharmacokinetic profile of the dual-target binding ligands. Uptake in the tumor, blood, and kidneys is plotted in Figure 6. The highest tumor uptake is observed for ${ }^{131}$ I-RPS001, and the kinetics of tumor washout are similar for the 3 compounds with lower affinity for albumin (Fig. 6A). The level of activity in the tumor remains most steady in the 2 ligands with highest albumin binding (Fig. 6A). Striking differences are apparent in the rate of blood clearance among the various compounds studied, with ${ }^{131}$ I-RPS-005 and ${ }^{131}$ I-RPS020, both having high affinity for albumin, showing minimal blood clearance over $48 \mathrm{~h}$ and beyond (Fig. 6B). The clearance kinetics of ${ }^{131}$ I-RPS-001, ${ }^{131}$ I-RPS-022, and ${ }^{131}$ I-RPS-027 reflect their relative affinities for albumin, with ${ }^{131}$ I-RPS-022 clearing most quickly and ${ }^{131}$ I-RPS-027 clearing most gradually. Blood curves showed a rapid initial distribution phase followed by a slower elimination phase. In this model, the half-life for the distribution phase was $2.17,2.1$, and $3.15 \mathrm{~h}$ for 

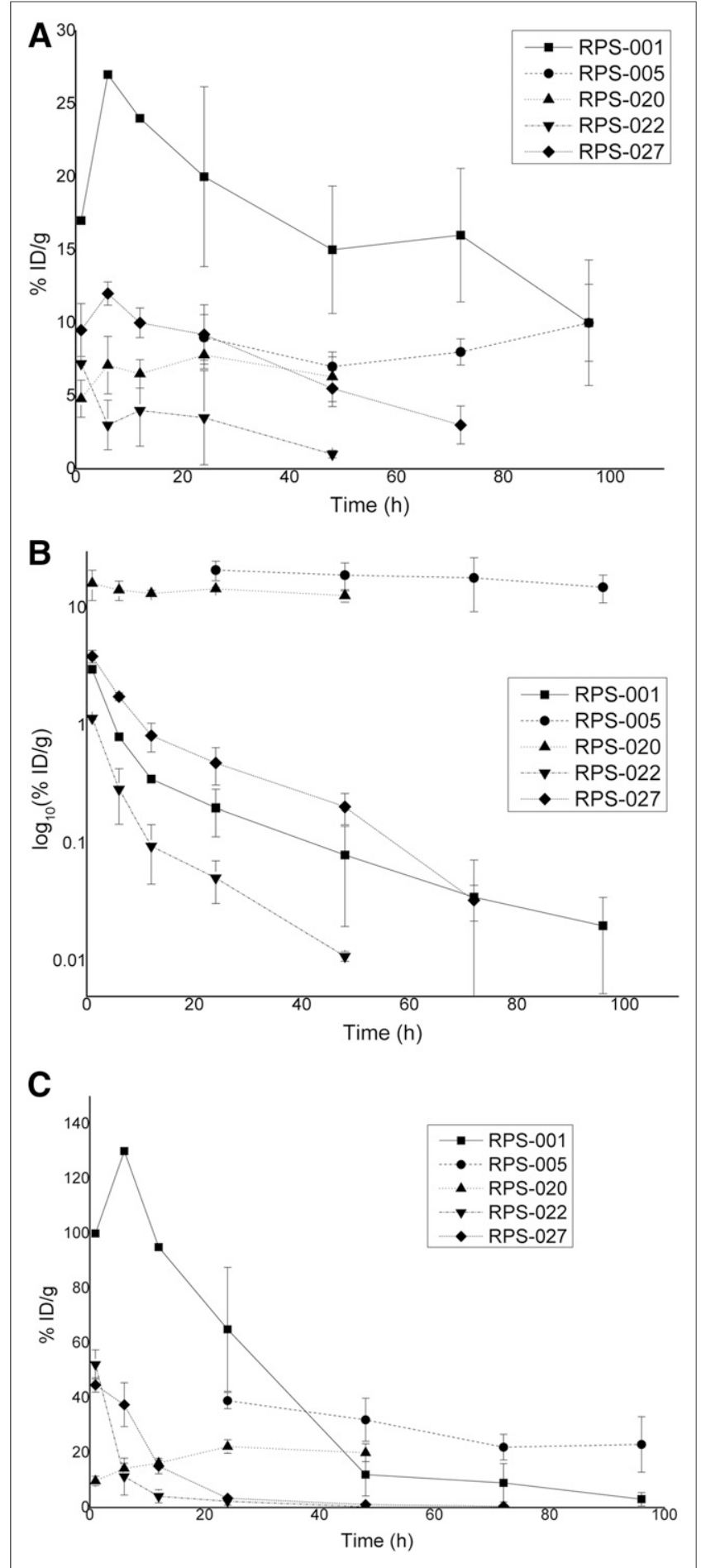

FIGURE 6. Time-activity curves for blood (A), tumor (B), and kidneys (C) derived from biodistribution data. Error bars represent SD of measured values.

${ }^{131}$ I-RPS-001, ${ }^{131}$ I-RPS-022, and ${ }^{131}$ I-RPS-027, respectively, whereas the corresponding half-life for the elimination phase was 20.38, 17.3, and $21.66 \mathrm{~h}$. In comparison, the half-lives for distribution and elimination phases for ${ }^{131}$ I-RPS-020 were 3.85 and 3,300 h, respectively.

Absolute kidney uptake of RPS-027 and RPS-022 was dramatically reduced compared with RPS-001 at all time points studied.
Kidney clearance is approximately described by an exponential decay function for ${ }^{131}$ I-RPS-001, ${ }^{131}$ I-RPS-022, and ${ }^{131}$ I-RPS-027 (Fig. 6C). In contrast, ${ }^{131} \mathrm{I}-\mathrm{RPS}-005$ and ${ }^{131} \mathrm{I}-020$ show prolonged retention and much flatter clearance curves. Tumor-to-kidney and tumor-to-blood ratios were calculated as a function of time. The tumor-to-kidney ratio of ${ }^{131}$ I-RPS-027 reached approximately 3 by $24 \mathrm{~h}$ and continued to increase significantly with time (Fig. 7A). In comparison, the tumor-to-kidney ratio of ${ }^{131}$ I-RPS-001 did not reach 3 until nearly $96 \mathrm{~h}$ after injection. The tumor-to-kidney ratio of ${ }^{131}$ I-RPS-022 also increased rapidly, particularly at earlier time points and significantly by $12 \mathrm{~h}$ after injection $(>1)$, but this was driven more by rapid kidney clearance than by tumor uptake. The tumor-to-blood ratio of ${ }^{131}$ I-RPS-027 was lower than ${ }^{131} \mathrm{I}$ RPS-001 (Fig. 7B), predominantly reflecting enhanced albumin binding.

\section{DISCUSSION}

In the absence of stable isotopes of astatine, iodine has been proposed as a useful surrogate for drug development and for predicting radiation dosimetry. Recent work has confirmed that the pharmacokinetics of a small-molecule PSMA inhibitor, ${ }^{131} \mathrm{I}-\mathrm{DCIBzL}$, and its astatinated analog (2S)-2-(3-(1-carboxy-5(4-211 At-astatobenzamido)pentyl)ureido)-pentanedioic acid ( $\left.{ }^{211} \mathrm{At}-6\right)$ (38) were similar in a preclinical prostate cancer model, although small but statistically significant differences were observed. These differences included an apparent decrease in PSMA affinity for the astatinated analog, as evidenced by a 10\%-30\% reduction of uptake in PSMA-expressing tissues, and an apparent increase in blood binding (38).

Modulation of blood binding has been demonstrated preclinically to increase tumor uptake and decrease kidney uptake $(28,39)$ without causing a corresponding increase in hematologic toxicity. In a recent study of DOTA-conjugated albumin labeled with the $\alpha$-emitter ${ }^{213} \mathrm{Bi}$ (half-life $=45 \mathrm{~min}$ ), doses up to $3.7 \mathrm{MBq}$ could be administered to mice safely, with no plasma enzyme modifications or histologic abnormalities detected over $1 \mathrm{y}$ of observation (40). This is in sharp contrast to recently reported findings of a small-molecule PSMA inhibitor labeled with ${ }^{211} \mathrm{At}$ (half-life $=7.2 \mathrm{~h}$ ) in which the maximum tolerated single dose in immunocompetent mice was 2 orders of magnitude lower, at $0.037 \mathrm{MBq}$, due to severe, irreversible renal toxicity (38). High kidney uptake was also observed for ${ }^{131}$ I-MIP-1095 in mice, although this did not prove to be dose-limiting during initial clinical evaluation in humans of a single therapy cycle (2). In this context, the increased blood-pool half-life of ${ }^{131} \mathrm{I}-$ RPS-027 and significantly lower renal uptake and retention, relative to ${ }^{131}$ I-MIP-1095, facilitated by enhanced binding to albumin, represent an important component to an increased radiotherapeutic index.

It is likely that the reduction in kidney uptake on albumin binding is due to a decrease in the rate of filtration and reabsorption in proximal renal tubules $(41,42)$. Because PSMA is also expressed in proximal renal tubules $(43,44)$, it is possible that decreases in kidney uptake might be a function of reduced affinity for PSMA. However, the observation that RPS-027 $\left(\mathrm{IC}_{50}=\right.$ $15 \mathrm{nM}$ ) shows higher initial uptake than the strongly albuminassociating RPS-020 ( $\left.\mathrm{IC}_{50}=8 \mathrm{nM}\right)$ and lower initial uptake than the weakly associating RPS- $022\left(\mathrm{IC}_{50}=10 \mathrm{nM}\right)$ suggests that differences in kidney uptake are indeed mediated by albumin binding. 


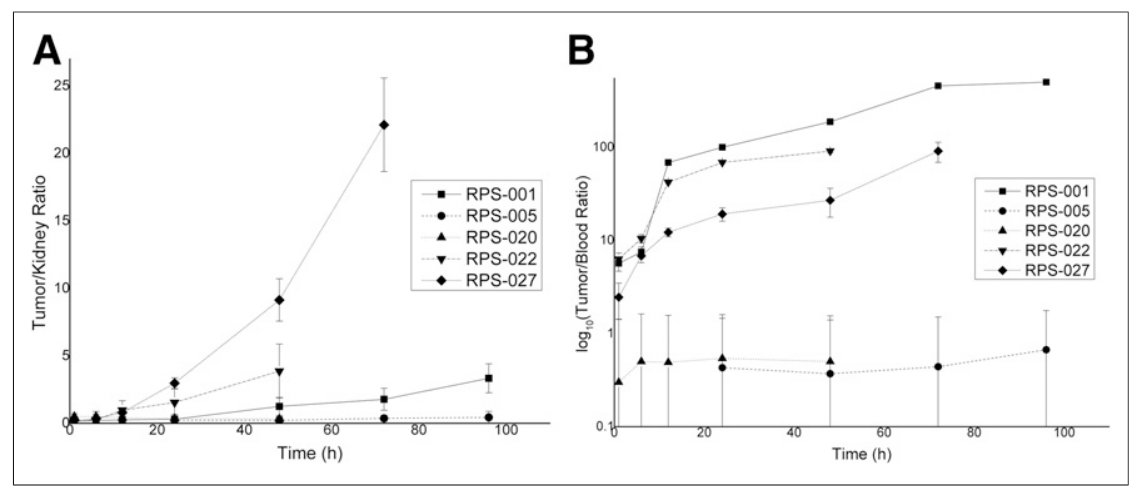

FIGURE 7. Tumor-to-background ratios show effect of enhanced albumin binding. Error bars were computed from SD of measured values. (A) Tumor-to-kidney ratio. (B) Tumor-to-blood ratio.

study on dual-target binding ligands with unequal affinity for both PSMA and serum albumin. ${ }^{131}$ I-RPS-027 was prepared in $49.5 \%$ radiochemical yield from its organostannane precursor and showed good tumor uptake and retention in a $\mathrm{LNCaP}$ xenograft model up to $72 \mathrm{~h}$ after injection. Rapid washout was observed in other tissues, including the kidneys. These pharmacokinetics appear to be a consequence of an appropriate level of binding to albumin $(\mathrm{Kd}=11 \mu \mathrm{M})$ that extends blood clearance, increases tumor uptake by increasing the number of passes through the tumor bed, and reduces kidney uptake by decreasing renal filtration and reuptake. With a

In comparison to ${ }^{131} \mathrm{I}-\mathrm{MIP}-1095$ (45), ${ }^{131} \mathrm{I}-\mathrm{DCIBzL}$ (46), and ${ }^{211}$ At-6 (38), ${ }^{131}$ I-RPS-027 shows considerably lower kidney uptake at all time points from 1 to $72 \mathrm{~h}$ after injection. The difference in uptake between ${ }^{131} \mathrm{I}$-MIP-1095 $\left({ }^{131} \mathrm{I}\right.$-RPS-001) and ${ }^{131} \mathrm{I}$-RPS-027 at $24 \mathrm{~h}$ is 20 -fold. In the context of the dosimetry reported for MIP1095 in patients, the lower kidney uptake of ${ }^{131}$ I-RPS-027 projects to a significantly lower absorbed dose to the kidneys, and a reduced risk of relevant nephrotoxicity at therapeutic doses or under a multiple treatment cycle regime. Moreover, the tumor-to-kidney ratio for ${ }^{131} \mathrm{I}$-RPS- 027 is greater than 2 as soon as $18 \mathrm{~h}$ after injection, rises to 3 by $24 \mathrm{~h}$, and exceeds 7 by $72 \mathrm{~h}$. This compares favorably with ${ }^{211} \mathrm{At}-6$, and a projection of the comparison between ${ }^{131} \mathrm{I}$ DCIBzL and ${ }^{211}$ At- 6 to RPS-027 predicts that astatination will further increase the ratio (38). As such, the dose-limiting, irreversible nephrotoxicity of ${ }^{211}$ At- 6 might be resolved by ${ }^{211}$ At-RPS- 027 . ${ }^{131}$ I-RPS-022 also shows higher tumor-to-background ratios than ${ }^{131}$ I-RPS-001 and ${ }^{211}$ At- 6 at early time points, but this is driven by rapid excretion rather than by tumor uptake.

Dose-limiting toxicity to the salivary glands was observed for ${ }^{131}$ I-MIP-1095 (2). Moderate xerostomia has been reported for ${ }^{177} \mathrm{Lu}-\mathrm{PSMA}-617(3,6)$, but translation of this ligand to targeted $\alpha$-particle therapy as ${ }^{225}$ Ac-PSMA-617 led to severe and sustained xerostomia (18). It has been reported that PSMA is expressed at low levels in the parotid and lacrimal glands (47), and recent experiments have shown that 2-(phosphonomethyl)-pentandioic acid can be used to displace ${ }^{68} \mathrm{Ga}$-PSMA-HBED-CC from rat salivary glands (48). These findings suggest that uptake of radiopharmaceuticals in these structures is PSMA-mediated. Uniquely to the class of glutamateurea heterodimers found in most clinically investigated radiolabeled PSMA inhibitors, the fluorinated small-molecule PSMA inhibitor ${ }^{18}$ F-DCFBC showed tumor localization with moderately persistent blood-pool activity and a corresponding decrease in signal intensity in the salivary glands (49), suggesting that albumin-binding is a potential strategy for reducing salivary gland uptake that, a priori, does not rule out adequate tumor uptake in humans. Plasma binding has been demonstrated to reduce salivary gland uptake of cyclosporine (50) and warfarin (51) in rats. Because the small size of murine salivary glands precludes detection of uptake by imaging or biodistribution studies, work is ongoing to confirm the effect of modulated albumin binding on salivary gland uptake in larger animals.

\section{CONCLUSION}

A low-molecular-weight PSMA inhibitor $\left(\mathrm{IC}_{50}=15 \mathrm{nM}\right)$, RPS027, was developed as part of a structure-activity relationship highly favorable tumor-to-kidney ratio ( $>3$ by $24 \mathrm{~h}$ after injection), the projected dosimetry of RPS-027 compares favorably with MIP1095. Using iodine/radioiodine as a surrogate for the radiohalogen ${ }^{211}$ At, we propose dual-target binding ligands such as RPS-027 as a next-generation radiopharmaceutical for targeted $\alpha$-therapy of prostate cancer using ${ }^{211} \mathrm{At}$.

\section{DISCLOSURE}

No potential conflict of interest relevant to this article was reported.

\section{ACKNOWLEDGMENT}

We thank Dr. Yeona Kang for her assistance in determining blood clearance kinetics and statistical analysis.

\section{REFERENCES}

1. Strosberg JR, Wolin EM, Chasen B, et al. NETTER-1 phase III: progression-free survival, radiographic response, and preliminary overall survival results in patients with midgut neuroendocrine tumors treated with 177-Lu-dotatate [abstract]. J Clin Oncol. 2016;34(suppl 4S):194.

2. Zechmann CM, Afshar-Oromieh A, Armor T, et al. Radiation dosimetry and first therapy results with a ${ }^{124} \mathrm{I} /{ }^{131} \mathrm{I}$-labeled small molecule (MIP-1095) targeting PSMA for prostate cancer therapy. Eur J Nucl Med Mol Imaging. 2014;41:1280-1292.

3. Kratochwil C, Giesel FL, Stefanova M, et al. PSMA-Targeted radionuclide therapy of metastatic castration-resistant prostate cancer with ${ }^{177} \mathrm{Lu}$-labeled PSMA617. J Nucl Med. 2016;57:1170-1176.

4. Baum RP, Kulkarni HR, Schuchardt C, et al. Lutetium-177 PSMA radioligand therapy of metastatic castration-resistant prostate cancer: safety and efficacy. J Nucl Med. 2016;57:1006-1013.

5. Heck MM, Retz M, D’Alessandria C, et al. Systemic radioligand therapy with ${ }^{177} \mathrm{Lu}$ labeled prostate specific membrane antigen ligand for imaging and therapy in patients with metastatic castration resistant prostate cancer. J Urol. 2016;196:382-391.

6. Fendler WP, Reinhardt S, Ilhan H, et al. Preliminary experience with dosimetry, response and patient reported outcome after ${ }^{177} \mathrm{Lu}$-PSMA-617 therapy for metastatic castration-resistant prostate cancer. Oncotarget. 2017;8:3581-3590.

7. Rahbar K, Schmidt M, Heinzel A, et al. Response and tolerability of a single dose of ${ }^{177} \mathrm{Lu}$-PSMA-617 in patients with metastatic castration-resistant prostate cancer: a multicenter retrospective analysis. J Nucl Med. 2016;57:1334-1338.

8. Ahmadzadehfar H, Eppard E, Kürpig S, et al. Therapeutic response and side effects of repeated radioligand therapy with ${ }^{177} \mathrm{Lu}-\mathrm{PSMA}-\mathrm{DKFZ}-617$ of castrateresistant metastatic prostate cancer. Oncotarget. 2016;7:12477-12488.

9. Afshar-Oromieh A, Hetzheim H, Kratochwil C, et al. The theranostic PSMA ligand PSMA-617 in the diagnosis of prostate cancer by PET/CT: biodistribution in humans, radiation dosimetry, and first evaluation of tumor lesions. J Nucl Med. 2015;56:1697-1705.

10. Dreher MR, Liu W, Michelich CR, Dewhirst MW, Yuan F, Chilkoti A. Tumor vascular permeability, accumulation, and penetration of macromolecular drug carriers. J Natl Cancer Inst. 2006;98:335-344. 
11. Choi IK, Strauss R, Richter M, Yun CO, Lieber A. Strategies to increase drug penetration in solid tumors. Front Oncol. 2013;3:193.

12. Minchinton AI, Tannock IF. Drug penetration in solid tumours. Nat Rev Cancer. 2006;6:583-592.

13. Arveschoug AK, Kramer SM, Iversen P, Frøkiær J, Grønbæk H. Monitoring kidney function in neuroendocrine tumor patients treated with ${ }^{90}$ Y-DOTATOC: associations with risk factors. Curr Radiopharm. 2015;8:49-55.

14. Moll S, Nickeleit V, Mueller-Brand J, Brunner FP, Maecke HR, Mihatsch MJ. A new cause of renal thrombotic microangiopathy: yttrium 90-DOTATOC internal radiotherapy. Am J Kidney Dis. 2001;37:847-851.

15. Giralt S, Bensinger W, Goodman M, et al. ${ }^{166}$ Ho-DOTMP plus melphalan followed by peripheral blood stem cell transplantation in patients with multiple myeloma: results of two phase 1/2 trials. Blood. 2003;102:2684-2691.

16. Kratochwil C, Bruchertseifer F, Giesel FL, et al. ${ }^{225}$ Ac-PSMA-617 for PSMA targeting alpha-radiation therapy of patients with metastatic castration-resistant prostate cancer. J Nucl Med. 2016;57:1941-1944.

17. Kratochwil C, Giesel FL, Leotta K, et al. PMPA for nephroprotection in PSMA-targeted radionuclide therapy of prostate cancer. J Nucl Med. 2015; 56:293-298.

18. Chatalic KLS, Heskamp S, Konijnenberg M, et al. Towards personalized treatment of prostate cancer: PSMA I\&T, a promising prostate-specific membrane antigen-targeted theranostic agent. Theranostics. 2016;6:849-861.

19. Blakemore C, Jennett S. The Oxford Companion to the Body. New York, NY: Oxford University Press; 2001.

20. Vaidyanathan G, Zalutsky MR. Targeted therapy using alpha emitters. Phys Med Biol. 1996;41:1915-1931.

21. Andersen JT, Dalhus B, Viuff D, et al. Extending serum half-life of albumin by engineering neonatal Fc receptor (FcRn) binding. J Biol Chem. 2014;289:1349213502.

22. Jacobson O, Kiesewetter DO, Chen X. Albumin-binding Evans Blue derivatives for diagnostic imaging and production of long-acting therapeutics. Bioconjug Chem. 2016;27:2239-2247.

23. Liu Z, Chen X. Simple bioconjugate chemistry serves great clinical advances: albumin as a versatile platform for diagnosis and precision therapy. Chem Soc Rev. 2016;45:1432-1456.

24. Dennis MS, Jin H, Dugger D, et al. Imaging tumors with an albumin-binding Fab, a novel tumor-targeting agent. Cancer Res. 2007;67:254-261.

25. Koehler MF, Zobel K, Beresini MH, et al. Albumin affinity tags increase peptide half-life in vivo. Bioorg Med Chem Lett. 2002;12:2883-2886.

26. Kratz F. Albumin as a drug carrier: design of prodrugs, drug conjugates and nanoparticles. J Control Release. 2008;132:171-183.

27. Caravan P. Protein-targeted gadolinium-based magnetic resonance imaging (MRI) contrast agents: design and mechanism of action. Acc Chem Res. 2009; 42:851-862.

28. Müller C, Struthers H, Winiger C, Zhernosekov K, Schibli R. DOTA conjugate with an albumin-binding entity enables the first folic-acid-targeted ${ }^{177} \mathrm{Lu}$-radionuclide tumor therapy in mice. $J$ Nucl Med. 2013;54:124-131.

29. Wilbur DS. Enigmatic astatine. Nat Chem. 2013;5:246.

30. Talanov VS, Yordanov AT, Garmestani K, et al. Preparation and in vivo evaluation of novel linkers for ${ }^{211}$ At labeling of proteins. Nucl Med Biol. 2004;31:1061-1071.

31. Ayed T, Pilme J, Teze D, et al. At-211-labeled agents for alpha-immunotherapy: on the in vivo stability of astatine-agent bonds. Eur J Med Chem. 2016;116:156-164.

32. Chen J, Hage DS. Quantitative studies of allosteric effects by biointeraction chromatography: analysis of protein binding for low-solubility drugs. Anal Chem. 2006;78:2672-2683.
33. Matsuda R, Anguizola J, Hoy KS, Hage DS. Analysis of drug-protein interactions by high-performance affinity chromatography: interactions of sulfonyl urea drugs with normal and glycated human serum albumin. Methods Mol Biol. 2015;1286:255-277.

34. Zheng X, Podariu M, Bi C, Hage DS. Development of enhanced capacity affinity microcolumn by using a hybrid of protein cross-linking/modification and immobilization. J Chromatogr A. 2015;1400:82-90.

35. Joseph KS, Hage DS. The effects of glycation on the binding of human serum albumin to warfarin and L-tryptophan. J Pharm Biomed Anal. 2010;53:811-818.

36. Kelly J, Amor-Coarasa A, Nikolopoulou A, et al. Synthesis and pre-clinical evaluation of a new class of high-affinity ${ }^{18} \mathrm{~F}$-labeled PSMA ligands for detection of prostate cancer by PET imaging. Eur J Nucl Med Mol Imaging. 2017;44:647-661.

37. Hillier SM, Maresca KP, Femia FJ, et al. Preclinical evaluation of novel glutamateurea-lysine analogs that target prostate specific membrane antigen as molecular imaging pharmaceuticals for prostate cancer. Cancer Res. 2009;69:6932-6940.

38. Kiess AP, Minn I, Vaidyanathan G, et al. (2S)-2-(3-(1-carboxy-5-(4-[ ${ }^{211}$ At]astatobenzamido)pentyl)ureido)-pentanedioic acid for PSMA-targeted $\alpha$-particle radiopharmaceutical therapy. J Nucl Med. 2016;57:1569-1575.

39. Tolmachev V, Orlova A, Pehrson R, et al. Radionuclide therapy of HER2-positive microxenografts using a ${ }^{177} \mathrm{Lu}$-labeled HER2-specific affibody molecule. Cancer Res. 2007;67:2773-2782.

40. Dorso L, Bigot-Corbel E, Abadie J, et al. Long-term toxicity of ${ }^{213} \mathrm{Bi}$-labelled BSA in mice. PLoS One. 2016;11:e0151330.

41. Vegt E, van Eerd JEM, Eek A, et al. Reducing renal uptake of radiolabeled peptides using albumin fragments. J Nucl Med. 2008;49:1506-1511.

42. Dennis MS, Zhang M, Meng YG, et al. Albumin binding as a general strategy for improving the pharmacokinetics of proteins. J Biol Chem. 2002;277:35035-35043.

43. Silver DA, Pellicer I, Fair WR, Heston WDW, Cordon-Cardo C. Prostate-specific membrane antigen expression in normal and malignant human tissues. Clin Cancer Res. 1997;3:81-85.

44. Baccala A, Sercia L, Li J, Heston W, Zhou M. Expression of prostate-specific membrane antigen in tumor-associated neovasculature of renal neoplasms. Urology. 2007;70:385-390.

45. Hillier S, Rubino K, Maresca K, et al. $\left[{ }^{131} \mathrm{I}\right] \mathrm{MIP}-1466$, a small molecule prostatespecific membrane antigen (PSMA) inhibitor for targeted radiotherapy of prostate cancer (PCa) [abstract]. J Nucl Med. 2012;53(suppl 1):170.

46. Chen Y, Foss CA, Byun Y, et al. Radiohalogenated prostate-specific membrane antigen (PSMA)-based ureas as imaging agents for prostate cancer. J Med Chem. 2008;51:7933-7943.

47. O'Keefe DS, Bacich DJ, Heston WDW. Comparative analysis of prostate-specific membrane antigen (PSMA) versus a prostate-specific membrane antigen-like gene. Prostate. 2004;58:200-210.

48. Wüstemann T, Nikolopoulou A, Amor-Coarasa A, et al. Protecting salivary glands: displacement of off-target bound prostate-specific membrane antigen ligands [abstract]. Eur J Nucl Med Mol Imaging. 2016;43(suppl 1):S15.

49. Cho SY, Gage KL, Mease RC, et al. Biodistribution, tumor detection, and radiation dosimetry of ${ }^{18} \mathrm{~F}$-DCFBC, a low-molecular-weight inhibitor of prostatespecific membrane antigen, in patients with metastatic prostate cancer. $J$ Nucl Med. 2012;53:1883-1891.

50. Lemaire M, Pardridge WM, Chaudhuri G. Influence of blood components on the tissue uptake indices of cyclosporin in rats. J Pharmacol Exp Ther. 1988; 244:740-743.

51. Urien S, Morin D, Tillement JP. Effect of alpha-1-acid glycoprotein, albumin and palmitic acid on the brain and salivary gland extraction of warfarin in rats. J Pharmacol Exp Ther. 1989;248:781-785. 